\title{
TOWARDS AN INTEGRATED SIMULATION AND VIRTUAL COMMISSIONING ENVIRONMENT FOR CONTROLS OF MATERIAL HANDLING SYSTEMS
}

\author{
Stephan Seidel \\ Ulrich Donath \\ Juergen Haufe \\ Fraunhofer Institute for Integrated Circuits \\ Design Automation Division \\ Zeunerstrasse 38 \\ D-01069 Dresden, GERMANY
}

\begin{abstract}
Modern material handling systems (MHS) are complex systems which are controlled by various control units on different automation levels. The design of the MHS facility layout and the development of the control units require the application of different $\mathrm{CAE}$ tools but simulation and virtual commissioning does currently not play a significant role because there is no integrated simulation-based verification environment for all project stages and control levels available. This paper presents an approach towards an integrated simulation-based verification tool for all stages of an MHS project. During the first stage a material flow simulation of the plant model is conducted to analyze key performance indicators. This model is reused to test and verify the function of control units such as material flow controllers or programmable logic controllers. An automatic equivalence checking tool identifies differences between simulation runs. An important benefit: One common simulation model is used for all project stages.
\end{abstract}

\section{INTRODUCTION}

Control components of different control levels as shown in Figure 1 are interacting with each other in current state-of-the-art material handling systems. The development of such systems is an interdisciplinary process in which know-how from various domains such as electrical engineering, mechanical engineering as well as computer sciences and business administration is necessary. The separation of the control algorithms into different levels is typical for various domains and a strategies on organizing control algorithms by using object-oriented methods has been published by Cho, Smith and Wysk (1997) and Smith and Joshi (1995).

Throughout the facility planning stage of material handling systems many key performance indicators (KPI) such as throughput, utilization and path length can be obtained and analyzed by modeling and simulating the system. In addition routing and storage strategies can be evaluated and compared. As a result of the facility planning stage functional definitions and requirement specifications for the material flow controller (MFC) as well as the programmable logic controllers (PLC) can be obtained.

The implementation, test and verification of control programs for the different control levels are carried out separately from each other. Because of the lack of a common test bench the MFC and PLC programs are thus tested individually. A joint test in conjunction with the real-word instrumentation and control components can only be done on site and at a very late stage during the projects duration. The consequences of this approach are inconsistencies between the intended behavior, which is detailed in the functional specification and the implemented behavior of the control systems. As a result the commission- 
ing of the system will be delayed and non-scheduled downtimes due to programming errors lead to increased financial and prestige risks for customer and contractor.

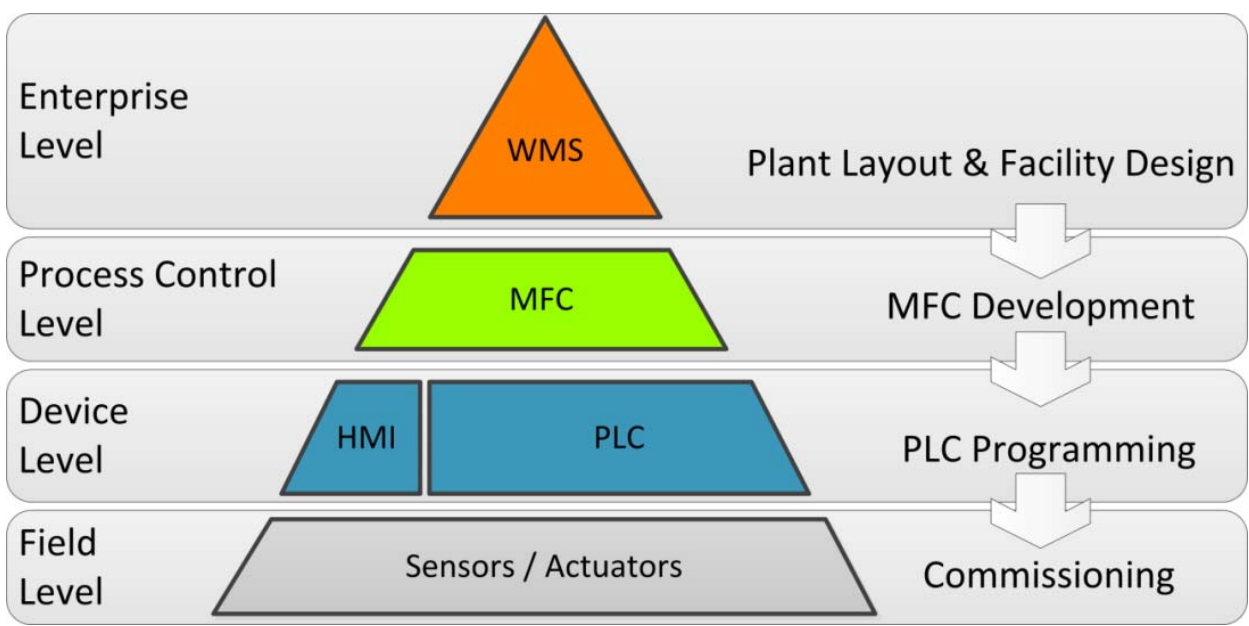

Figure 1: Control levels and corresponding project stages for material handling systems

\section{STATE-OF-THE-PRACTICE}

Computer-Aided-Engineering (CAE) tools are ubiquitous in planning and implementation of material handling systems. The layout is designed by using CAD software and is the basis for simulation tools which are employed to evaluate and confirm the systems KPI's. Several discrete event simulation (DES) tools such as PlantSimulation (Siemens AG 2012), AutoMOD (Applied Materials 2012) and Flexsim (Flexsim Software Products Inc. 2012) provide solutions for the following tasks:

- Design and evaluation of various layout options for a system

- Check of the system's overall function with regard to the material flow

- Determination of capacity limits and bottlenecks within the material handling system

- Comparison of systems from different vendors

- Development and test of storage and routing strategies

A consequent analysis of the simulation results and an iterative adjustment of the simulation model will result in an improved layout and a precise requirement specification which will comply with the defined performance figures. The simulation run may cover periods of weeks or months of operation time. The typical drawback with all aforementioned DES tools is that they do not provide connectivity with external control units such as MFC or PLC's. Communication with these controllers is therefore not possible and the material flow simulation is reduced to a verification of the system definition.

After the layout has been defined during the planning stage, the implementation stage commences in which the implementation of the MFC program and the PLC programs takes place. Several MFC tools exist on the market, which have to be adapted and configured to the required layout and subordinate controllers. It is very common that program test and commissioning are carried out manually without the support of simulation tools.

A simulation-based virtual commissioning of the different control levels has been discussed in scientific papers for years but is not entirely available due to different levels of abstraction and interfaces between the controller programs. Several papers (Schleipen et al. 2009 and Hoffmann et al. 2010) have been published on the subject of the virtual commissioning of process control systems. Schleipen et al. (2009) suggest a combined Software-in-the-Loop (SiL) and Hardware-in-the-Loop (HiL) approach, where the real SCADA system together with connected PLCs is tested against a virtual plant model. The main draw- 
back of this approach is the limitation of the plant model to the PLC level, i.e. the model provides only inputs and outputs (I/O) for the PLC. This approach requires existing PLC programs as well as existing SCADA projects which can only be commissioned together. Hoffmann et al. (2010) reviewed existing tool chains for virtual commissioning of manufacturing systems and observed that the benefit of virtual commissioning depends on certain factors. In general the required time for model creation and simulation has to be significantly shorter than the amount of time that is traditionally required for commissioning. The most important factor is the efficiency of the modeling process. In case a library exists, which contains the necessary model components, modeling will be generally shorter and more efficient than modeling from scratch. Therefore simulation is far more likely to be employed if such a library exists.

Typical simulation tools that enable the engineer to virtually commission the control systems are WinMOD (Mewes \& Partner GmbH 2012), Emulate3D (Emulate3D Ltd 2012), and Experior (Xcelgo $\mathrm{A} / \mathrm{S}$ 2012). With all of them a plant model of the material handling process is developed against which the PLC program is tested. The PLC which is either a software tool or a hardware module is commonly linked via OPC (OPC Foundation 2012), Ethernet or field bus systems to the simulation tool. However, these simulation tools do not provide MFC interfaces because they focus on the virtual commissioning of PLCs. This field-level focus also explains why many of these tools feature a physics engine which enables the simulation of physical phenomena like friction, gravitation or collisions. A physics engine is an impressive feature but also requires a lot of computing and slows the simulation down. Moreover these tools cannot be seen as typical DES tools that are employed for the planning and simulation of MHS or production systems on a larger scale. Due to the aforementioned effects, larger material handling systems cannot be simulated effectively.

The communication between MFC and one or more PLC's is usually tested in the office, although an elaborate test is only possible on site at the real-world material handling system. Controller software testing on site has several drawbacks, such as systems which may be still under construction not being fully equipped. In addition precious test time must be shared with other trades. The project's overall success is often threatened when only a very narrow time slot is reserved for testing at the end of the project due to delays. This is typical for modification, retrofit or enlargement projects where parts of the process remain in operation.

Virtual commissioning of a line head control including DES and PLCs is discussed in Bangsow (2012) and the conclusion drawn were very positive. The benefits from virtual commissioning are an improved communication with automation developers, a greater maturity of plans, specifications and automation programs and a significantly reduced commissioning time. The article expressed the desire to extend virtual commissioning beyond the PLC level to data acquisition and MES levels. This envisioned approach is fulfilled w.r.t. material handling systems by the verification environment which is discussed in this paper.

Suggestions for an integrated tool chain for all project stages (facility planning, implementation and virtual commissioning) have been made by Gutenschwager (2003) and a first approach is the model export-import function of the software tools TarakosVR and WinMOD. TarakosVR is a CAE tool for creating a virtual 3D model from CAD data whereas WinMOD is a PLC oriented simulation tool. There is no integrated tool chain that supports design and simulation from the first prototype model to the fully functional model, which is used for the virtual commissioning of process control level and device level controllers. Common causes for these shortcomings are missing interfaces between material flow simulation tools and MFC or PLC controllers, as well as not existent model libraries which are required for such a tool chain. 
Seidel, Donath, and Haufe

\section{A SIMULATION ENVIRONMENT FOR PERFORMANCE ANALYSIS, PROGRAM TEST AND VIRTUAL COMMISSIONING}

\subsection{Overview}

This paper introduces an approach towards a simulation-based verification environment. This environment combines the layout planning, performance analysis, program test and verification of material handling systems which until now have been separated into process control level and device level. It will be shown that the aforementioned shortcomings on implementation and test can be avoided by using our tool chain.

The fundamental idea for the verification environment is the iterative evaluation of all control programs with a single process model. This model is created once during the facility planning stage and is used afterwards for the verification of MFC and PLC.



Figure 2: Application scenarios for a simulation environment during different project stages

The common basis for this approach is a model library that supports all implementation stages. The library components are typically modules from material handling systems such as conveyors, lift- and turntables, or transfer carriages. These components should provide functionality for simulation with the 
process control level (MFC) as well as the device level (PLC). Each level interacts differently with the simulation model. The lowest level is the device level which communicates I/O's between controller and model. In order to achieve a fast and reliable communication the data transfer is direct and accurate with respect to the controller cycle. Bus systems that might be present in the real-world system have been intentionally omitted from the model to avoid unnecessary complications.

The process model is just like the real system divided into several components. Each component represents a part of the process and contains the plant model of their real-world counterpart. The plant model transports the items by using kinematic commands according to instructions given by the relevant control unit. Events are generated when a certain condition or position (e.g. a light barrier) has been reached or a timer has elapsed.

The functionality of the components is configurable. In other words, each component will feature a set of functions that are relevant for route calculation, update and processing of inputs and outputs and other control operations. These functions can be activated or deactivated with respect to the connected control level. If a PLC is connected, a typical conveyor component will have light barriers and a drive but no internal logic. When it is connected to a MFC, the same component must feature an internal logic that represents a control unit that sends and receives transport commands.

Another feature is the option to record the material flow through the model during all development stages and save the records into a database. The datasets are later on required to check the consistency of different simulation runs as shown in Figure 6. It is therefore feasible to compare the simulation results of all development stages.

\subsection{Stage 1: Facility Design, Plant Layout and Performance Analysis}

As already mentioned, the main purpose of DES and material flow simulation tools is to evaluate different plant layouts. By modeling different layouts or changing the parameter set of the layout, it is possible to establish typical KPI's for each simulation run. In addition various test cases may be created, which can represent typical sequences of transport transactions (succession of transport tasks) by generating the corresponding items at the model's item sources. The test cases are the basis for the consistency check between different simulation runs. With respect to the consistency within the simulation runs it is recommended to select one or more simulation runs from stage 1 and use these results as reference results with which other results can be verified.

The efficiency of the layout with respect to capacity and performance is assessed with the simulation, and the most effective or cost efficient layout is found even before the implementation commences. As a result of stage 1, the system layout is defined and important parameters such as storage capacity, conveyor speeds and routing algorithms are identified. This stage is in accordance with the common material flow simulation, and the plant model abstractly describes process control level and device level.

The models of the components (conveyor, lift table, etc.) used to make the plant model, contain functions for routing the items and for controlling the item movement as shown in Figure 3.

During simulation in this stage the simulation run speed can be increased so that a long period such as days or even weeks can be simulated in a relatively short time, which is typical for discrete-event material flow simulations.

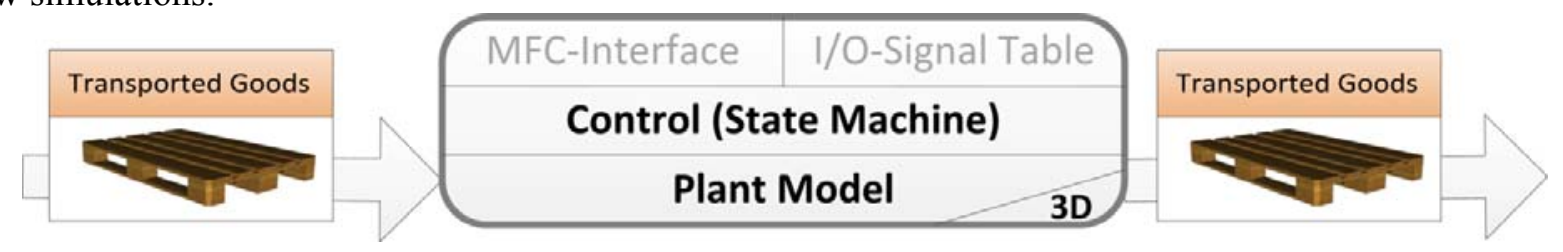

Figure 3: Structure of the Component Models during Project Stage 1 


\subsection{Stage 2: Implementation and Test of the Material Flow Controller}

The development of the material flow controller is based on the layout from stage 1, the simulated routing strategies, and the requirement specifications. Normally, the MFC is configured rather than programmed, but either way thorough testing or verification is needed.

The component models for simulation-based MFC verification used in the plant model still contain the device level algorithms until the internal routing functions are deactivated and an interface to the external MFC is activated. The routing of the items then becomes controlled by the external MFC application. All model components that handle items and require a routing decision will communicate with the MFC, which controls the material flow by sending transport tasks for each item to the simulation tool. Each component that handles an item will send a transport status update to the MFC as shown in Figure 4. This way the MFC is tested and virtually commissioned. All items can be reset in no time because the model is virtual, which is not easily done in the real-world. Testing is therefore much more efficient and less time consuming. In addition, the implementation of different routing strategies in the MFC can be simulated and analyzed based on KPI's such as efficiency and processing time.

Similarly to stage 1 , the flow of the items through the model, i.e. all transport transactions can be recorded and stored in a database. These recordings can be used for a post-simulative equivalence check. With this check, differences between the reference model from stage 1 and the MFC implementation from stage 2 can be detected.

The simulation's run speed can be adjusted similarly to stage 1. A possible bottleneck which may slow down the overall simulation speed, though, is the communication with the MFC.

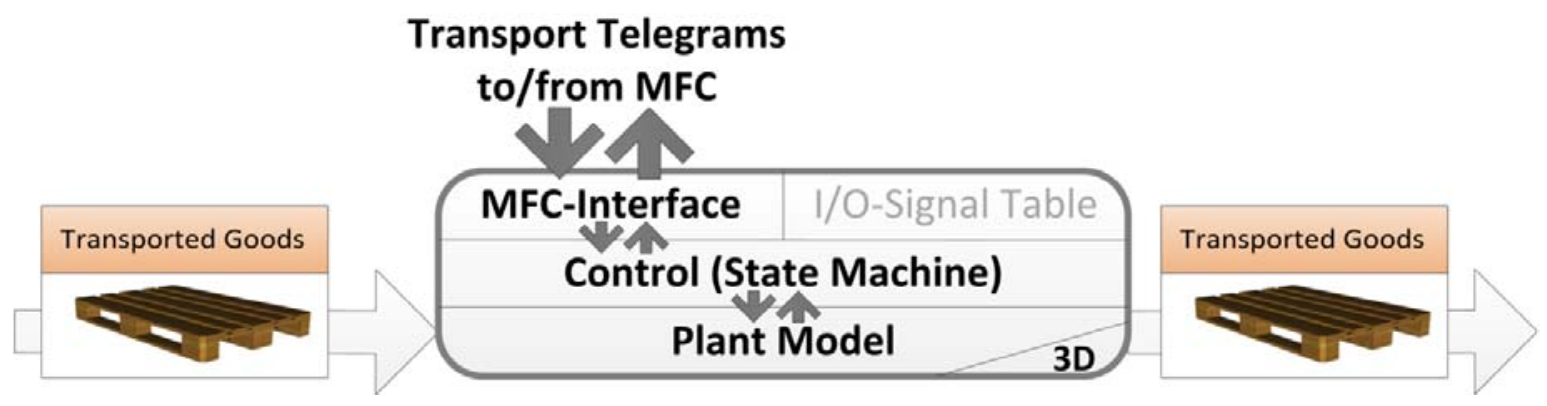

Figure 4: Structure of the Component Models during Project Stage 2

\subsection{Stage 3: Implementation and Test of the Programmable Logic Controller}

At this stage a control engineer develops the PLC program based on the requirements specification and the behavior of the reference model with the help of typical IDEs such as Siemens Step7 or CoDeSys. After the implementation of the control algorithms and functions into a PLC program, the co-simulation of model and software-PLC can commence. In other words, in stage 3 the PLC is running in a software-inthe-loop environment. The PLC program itself is being executed on a software PLC which emulates a hardware PLC. Therefore the PLC program requires no changes for running on a hardware PLC. In addition to the PLC program the device-level human-machine interface (HMI) can be developed, implemented, and tested. Usually this will be either a touch panel with a connection to the PLC or just a simulated runtime of this HMI. This enables the engineer not only to observe the process through the simulation visualization but also through the HMI which the process operator will use later on. Therefore this interface can be tested along with the PLC program.

At this stage the plant model and the relevant component models must be reconfigured. The reconfiguration consists of two steps. First, the components' internal control logic has to be deactivated because the PLC will process all input signals such as light barriers or position sensors, and set the output signals accordingly. . Second, the PLC's addresses (input, output, flag memory, data block, etc.) of the I/O signals have to be defined for each component. A signal table exists on each component and contains addresses and values for all corresponding PLC signals. It should be noted that a mixed component configu- 
ration within the plant model is possible. This enables the engineer to test the PLC code for certain components while others for which the PLC code is not ready yet are controlled by their own internal control logic.

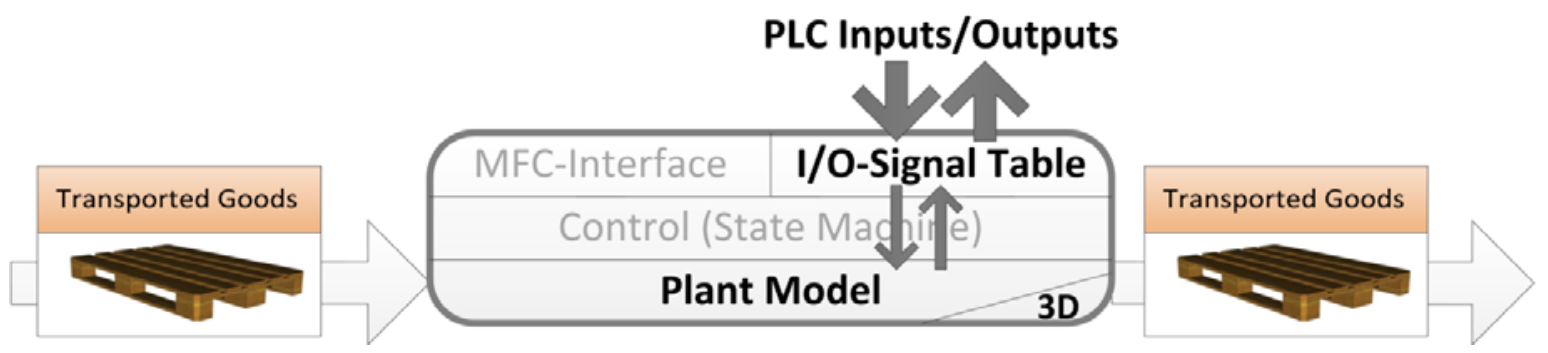

Figure 5: Structure of the Component Models during Project Stage 3

The component model will generate all input signals according to the position and speed of the items. Output signals from the PLC are analyzed and the item movement in the model (respectively kinematics) is set accordingly. This update procedure is executed cyclically. The update interval or rather simulation cycle time can be set to typical PLC cycle time values, e.g. $25 \mathrm{msec}$. It is the key factor for the resulting simulation performance. If the software PLC's controller cycle time is shorter than the simulation cycle time, the simulation can run even faster than real-time depending on the ratio of simulation cycle to controller cycle time. Because the component model is a discrete event model typical phenomena as described in Hoher et al. (2012) are avoided.

The PLC, which is a device level controller, will communicate with the plant model representing the field level through I/O's. The PLC can also be connected to the MFC. In this case transport task and status will be communicated with the MFC. Recording the material flow is also possible for the PLC cosimulation.

This stage correlates to a virtual commissioning of the PLC and connected HMI's. It enables the control engineer to test and evaluate the PLC program at a considerably earlier point during the project than was previously possible.

\subsection{Simulation Results Equivalence Check}

By examining the model's 3D-view the engineer has the advantage of a detailed overview of the whole process. All flow items can be followed on their way through the model. On the downside he is likely to lose track of a single item if there are hundreds or thousands of them flowing through the model. If he does lose track then differences in item routing may be overlooked by the engineer. This happens especially with large models or very long simulation periods.

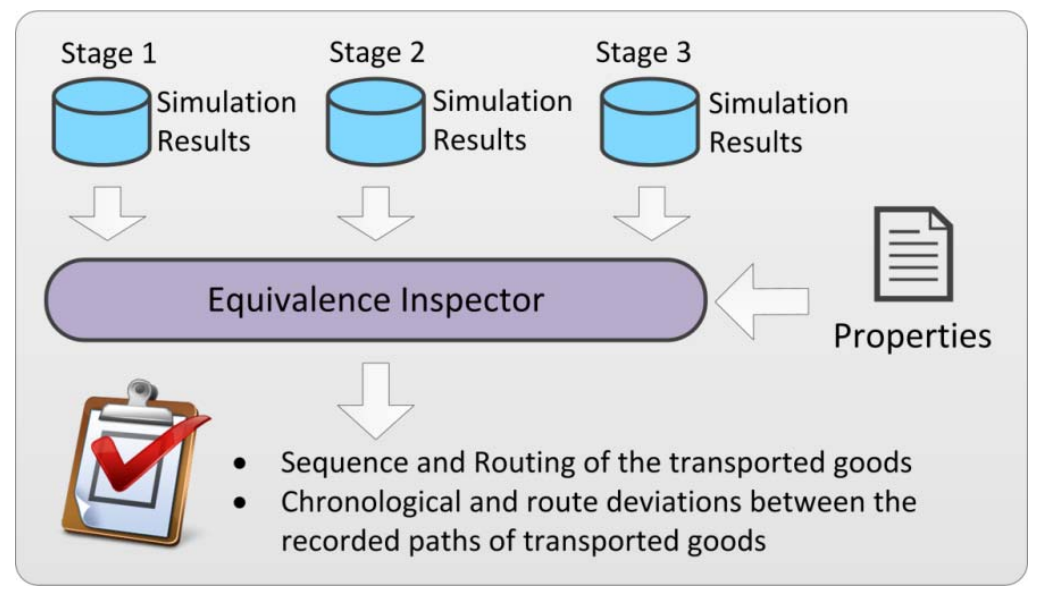

Figure 6: Simulation Results Equivalence Checking Tool 
To resolve this shortcoming of the simulation an automatic equivalency check has been implemented. As mentioned before, during each simulation run the material flow is recorded and the results are stored in a database. The record contains item ID, source, target and a time stamp. A new record is created as soon as an item is transferred from one model component to another. The automatic equivalency check compares the records of the material flow from two simulation runs. Typically one record will be a reference to be compared with the record from the newest run. For example, the reference result can originate from the reference model of stage 1, while the other record is from a PLC co-simulation run of stage 3.

Table 1: Example for equivalence check results showing that items $7 \& 10$ took different routes

\begin{tabular}{|c|c|c|c|c|c|c|c|}
\hline \multirow[b]{2}{*}{ Item } & \multirow[b]{2}{*}{$\begin{array}{c}\text { Path } \\
\text { different? }\end{array}$} & \multicolumn{3}{|c|}{ Difference details: $^{1}$} & \multicolumn{3}{|c|}{ Throughput time } \\
\hline & & $\begin{array}{c}\text { Object where } \\
\text { first difference oc- } \\
\text { curred? }\end{array}$ & $\begin{array}{c}\text { Object where } \\
\text { last difference oc- } \\
\text { curred? }\end{array}$ & $\begin{array}{c}\text { Different tar- } \\
\text { get? }\end{array}$ & $\begin{array}{c}\text { Run } \\
1\end{array}$ & $\begin{array}{c}\text { Run } \\
2\end{array}$ & $\begin{array}{l}\text { Not simi- } \\
\text { lar? }^{2}\end{array}$ \\
\hline 1 & & & & & $96 \mathrm{~s}$ & $96 \mathrm{~s}$ & \\
\hline 2 & & & & & $42 \mathrm{~s}$ & $42 \mathrm{~s}$ & \\
\hline 3 & & & & & $48 \mathrm{~s}$ & $48 \mathrm{~s}$ & \\
\hline 4 & & & & & $36 \mathrm{~s}$ & $36 \mathrm{~s}$ & \\
\hline 5 & & & & & $48 \mathrm{~s}$ & $48 \mathrm{~s}$ & \\
\hline 6 & & & & & $54 \mathrm{~s}$ & $54 \mathrm{~s}$ & \\
\hline 7 & $\bar{X}$ & Conveyor2 & Conveyor5 & & $36 \mathrm{~s}$ & $58 \mathrm{~s}$ & \\
\hline 8 & & & & & $40 \mathrm{~s}$ & $36 \mathrm{~s}$ & \\
\hline 9 & & & & & $46 \mathrm{~s}$ & $56 \mathrm{~s}$ & \\
\hline 10 & $\mathrm{X}$ & Conveyor 2 & Conveyor5 & & $52 \mathrm{~s}$ & $36 \mathrm{~s}$ & \\
\hline 11 & & & & & $58 \mathrm{~s}$ & $58 \mathrm{~s}$ & \\
\hline 12 & & & & & $36 \mathrm{~s}$ & $36 \mathrm{~s}$ & \\
\hline 13 & & & & & $58 \mathrm{~s}$ & $58 \mathrm{~s}$ & \\
\hline 14 & & & & & $64 \mathrm{~s}$ & $64 \mathrm{~s}$ & \\
\hline 15 & & & & & $68 \mathrm{~s}$ & $68 \mathrm{~s}$ & \\
\hline
\end{tabular}

There are a few prerequisites for using the checking tool. Most importantly the plant model layouts must be the same and the names from all components should be identical. In addition the sequence of items entering the model and their IDs have to be identical. If this is true, the check tool shown in Figure 6 will determine if each item in either simulation run has taken exactly the same path through the model. As shown in Table 1 deviations in the route will be detected and highlighted. Chronological and sequential deviations are also detected. Chronological deviations can occur if items take too long to pass through the model so that they arrive significantly earlier or later in the reference record. Because the two simulations runs will rarely be identical a tolerance band for the event times can be defined. Sequential deviations can happen if there are components in a model which merges separate material flows. The sequence of the items after the merge component may differ between the simulation runs even if all items take the same route because of variations, such as slightly different conveying times.

A report containing the equivalence check results is generated and provided as a table of highlighted deviations. This will enable the engineer to easily detect the component that causes the deviations and analyze the relevant control program code. An example report is shown in Table 1. 
By comparing the simulation results of the different implementation stages it will be ensured that the plant behavior introduced by the external control programs of MFC and PLC is equal to the reference model with respect to the relevant test case.

\subsection{Virtual Commissioning of the Control System}

At the end of the last stage all control programs have been fully implemented. Stage 3 is therefore relevant for the test and evaluation of the PLC program. By connecting the PLC with the MFC, all control systems and their interactions can be tested together. This way a virtual commissioning of MFC, PLC and HMI is feasible. This has several positive effects. Overall software quality will be improved, because evaluation and testing begins much earlier than previously possible. The contractor will install tested software, therefore the amount and duration of software tests on-site are reduced significantly. Another benefit is the ability to discuss and show the function of the control programs and interfaces to customer while using a virtual instance of the future facility. The customer's employees, who will later run the plant, can be trained in the operation of the plant by means of its virtual counterpart. Operator interactions are demonstrated and can be easily repeated. Potentially dangerous plant conditions can be simulated as well, which would not be possible at the real plant. Furthermore, future extensions of the plant can be modeled, simulated and analyzed by using and extending the model.

\section{PROJECT PARTNERS AND COMPONENTS}

The proposed simulation-based verification environment, which will be called Flexsim IL, has been developed by Fraunhofer IIS in collaboration with Dresden Informatik GmbH and was funded by the Federal Ministry of Economics and Technology. Environment elements are a process component library that supports three configurations for the aforementioned three stages for facility planning, process control level (MFC) and device level (PLC) test and verification. Other elements include an interface for communication with the software PLC and the equivalency checking tool.

The discrete-event material flow simulator, Flexsim, is the key element of the verification environment. External tools such as the MFC program (Dresden Informatik 2012) or the PLC control program are parts of the environment that are developed with external IDE's but evaluated and tested with the simulation environment. The software PLC S7-Simulation is a product by IBHsoftec GmbH (IBHsoftec GmbH 2012) which simulates a Siemens S7-416 PLC (Siemens AG 2012).

\section{RESULTS AND OUTLOOK}

The verification environment was tested by project partner Dresden Informatik within an industry project where it was employed as a test and verification tool for the PLC and MFC programs. In this project an existing material handling system was retrofitted with new PLC's (older Siemens S5 PLCs were exchanged with newer S7 PLCs) and the old MFC was replaced.

Results show that developed verification environment was perfectly suited for the virtual commissioning of the PLC program. During these simulation runs the PLC (around 500 I/O signals) was communicating transport status to and getting transport tasks from the developed MFC which was thus evaluated and tested as well. Hence PLC, HMI and MFC were virtually commissioned.

The additional work of modeling the material handling process in the simulator was compensated with a reduced commissioning stage on-site. Intense testing of PLC and MFC programs significantly boosted the software's maturity and reduced the required time on-site to $25 \%$ of the planned time.

The component models are applicable in all project stages. The model of the MHS process consists of multiple component models that are interconnected and configurable according to each stage. The use of different component configurations in one plant model is practicable, and one part of the model can thus be remotely controlled by an external PLC or MFC, whereas other parts are controlled by their internal logic. 
During the first stage, facility planning and layout performance analysis, the component behavior is simplified to the logistic transactions in which the routing is managed by local algorithms or a global routing table. The control development for the process control level (MFC) is the second stage and the routing is controlled by transport telegrams which are communicated with an external MFC. The third and last stage is the control program development on device level (PLC). During this stage the components will emulate the sensors (light barriers) and the behavior of the actuators (conveyor drives) on field level. By analyzing and comparing the simulation results from stage 1 to stage 3 , the behavior equivalency of the developed control programs can be assessed.

Simulation in stage 1 and 2 can be accelerated almost without limit. Limiting factors are only model size and the hardware on which the simulation is executed. In stage 2 the reaction time of the MFC is a limiting factor that has to be considered. Simulation speed in stage 3 is mainly affected by the ratio of simulation cycle time and controller cycle time. In the test case, the controller cycle is 3 times faster than the simulation's cycle time $(30 \mathrm{~ms})$. As a result, the simulation speed can be accelerated to around 3 times faster than real time.

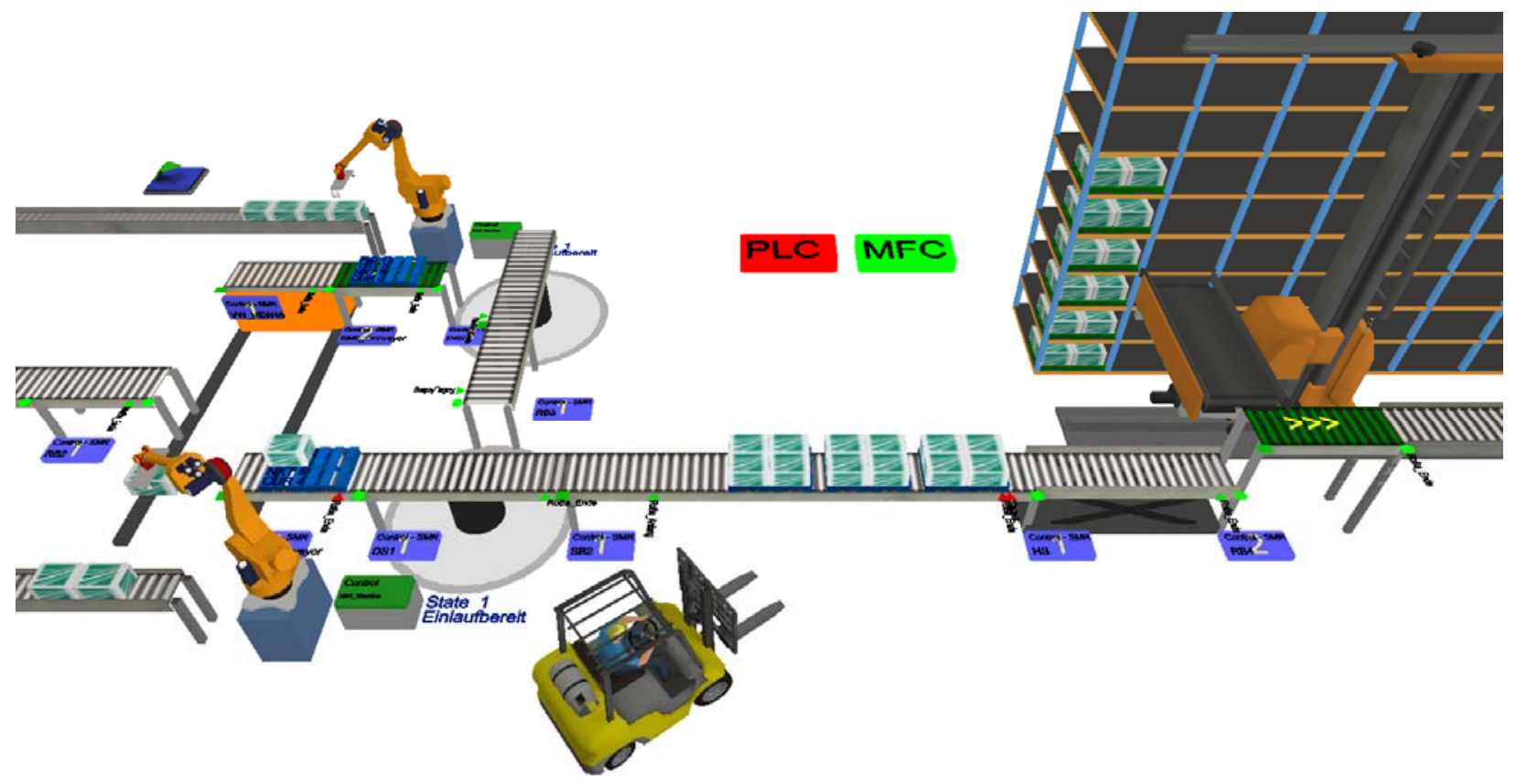

Figure 7: 3D view of an material handling system

After very positive project results the simulation and verification environment will be further developed. The component library has to be expanded to comprise of more standard material handling components, such as automatic storage and retrieval systems (ASRS) and additional conveyor components. New interfaces have to be added to the environment that will provide connectivity to additional software PLCs and also hardware PLCs, which will result in the ability to run HiL simulations.

Apart from planning, development, simulation-based verification and virtual commissioning the simulation and verification tool can also be employed during project acquisition or for operator training. The 3D view, as shown in Figure 7, is a very powerful tool for use in demonstrators and showcases. Feasibility studies for plant retrofits and extensions as well as performance analysis of existing plants are additional fields of application.

Dresden Informatik intends to conduct all future projects with the application of the developed simulation tool, especially with respect to the engineering of MFC and PLC programs. By doing so, the software maturity will be increased while planning and commissioning times will be significantly reduced. 
In the future the authors intend to merge the formal verification methodology for MHS into a common verification environment. The first results of formal verification of MHS were discussed by Klotz et. al. (2011) and show certain benefits that simulation-based verification cannot achieve.

\section{REFERENCES}

Applied Materials Inc., AutoMod web page,

http://appliedmaterials.com/services-software/library/applied-automod Accessed April 4, 2012.

Bangsow, S., Günther, U. 2012. "Creating a Model for Virtual Commissioning of a Line Head Control Using Discrete Event Simulation” In Use Cases of Discrete Event Simulation, Edited by S. Bangsow, $117-130$. Berlin, Heidelberg: Springer Verlag.

Cho, H., Smith, J. S., and Wysk, R. A., "A Robust Workstation Controller for Computer Integrated Manufacturing: Experimental Design and Results," In Production Planning and Control, Vol. 8, No. 6, 1997, pp.597-607.

Dresden Informatik, DiMFR web page, http://www.dresden-informatik.de/produkte/mfc_neu_EN.html Accessed April 4, 2012.

Emulate3D Ltd. 2012 . Emulate3D Controls Testing. http://www.simplan.de/images/stories/download/ENG/Emulate3DControls_Flyer.pdf, Accessed April 4, 2012.

Flexsim Software Products Inc. 2012. Flexsim Simulation Software. http://www.flexsim.com/flexsim/ Accessed April 4, 2012.

Gutenschwager, K., Kemper, J. 2003., "Neue Potentiale in der Logistiksimulation" In A\&D Kompendium 2003, 56-58. publish-industry Verlag GmbH.

Hoffmann, P., Schumann, R., Maksoud, T. M. A., and Premier, G. C. 2010., "Virtual Commissioning of Manufacturing Systems - A Review and new Approaches for Simplification" In Proceedings 24th Eu ropean Conference on Modelling and Simulation, Edited by A. Bargiela, S. A. Ali, D. Crowley, and E. J. H. Kerckhoffs, 175-181. European Council for Modelling and Simulation.

Hoher, S., Schindler, P., Göttlich, S., Schleper, V., and Röck, S., 2012, „System Dynamic Models and Real-time Simulation of Complex Material Flow Systems" In Enabling Manufacturing competitive ness and economic sustainability 2012, Part 3, Edited by H. A. ElMaraghy, 316-321. Berlin Heidelberg: Springer Verlag.

IBHsoftec GmbH. 2012."SoftPLC S7-416". http://www.ibhsoftec.com Accessed April 4, 2012.

Klotz, T., Straube, B., Fordran, E., Haufe, J., Schulze, F., Turek, K., and Schmidt., T. 2011 "An approach to the verification of material handling systems," In Emerging Technologies \& Factory Automation (ETFA), 2011 IEEE 16th Conference on, Institute of Electrical and Electronics Engineers.

Mewes \& Partner GmbH. 2010. WinMOD Report 09.31 - Virtual Commissioning with Material Flow Simulation. http://www.winmod.de/en/uploads/winmod_report_09.31_WM-SIMLINE.pdf . Accessed April 4, 2012.

OPC Foundation, About OPC - What is OPC? web page, http://www.opcfoundation.org/Default.aspx/01_about/01_whatis.asp?MID=AboutOPC Accessed April 5, 2012.

Schleipen, M., Sauer, O., Friess, N., Braun, L., and Shakerian, K. 2009 „Production Monitoring and Control Systems within the Digital Factory" In Proceedings of the 6th International Conference on Digital Enterprise Technology, Edited by G. Q. Huang, K. L. Mak, and P. G. Maropolous, 711-724. Berlin Heidelberg: Springer Verlag.

Siemens AG, PlantSimulation web page, http://www.plm.automation.siemens.com/en_us/products/tecnomatix/plant_design/plant_simulation.s html Accessed April 4, 2012.

Siemens AG, SIMATIC S7-400 Standard CPUs web page, http://www.automation.siemens.com Accessed April 4, 2012. 
Smith, J.S. and Joshi, S.B., “A Shop Floor Controller Class for Computer Integrated Manufacturing," In International Journal of Computer Integrated Manufacturing, Vol. 8, No. 5, September-October 1995, pp. 327-339.

Tarakos GmbH 2008. taraVRbuilder - 3D planning tool for a time based simulation. http://www.tarakos.com/documents/Builder en.pdf Accessed April 4, 2012.

Xcelgo A/S. 2012, "Experior PLC Tester”, http://www.simcon.dk, Accessed April 4, 2012.

\section{AUTHOR BIOGRAPHIES}

STEPHAN SEIDEL received his diploma in Electrical Engineering in 2003 from Dresden University of Technology. Thereafter he joined the Vinci Energies subdivision Actemium as a project engineer and was responsible for the implementation of control program and human interface projects for chemical distributions system in semiconductor fabs. In 2008 he joined Fraunhofer IIS EAS as research assistant and concentrates on the model-based development of automation systems as well as the virtual commissioning of material handling systems. His email address is stephan.seidel@eas.iis.fraunhofer.de.

ULRICH DONATH received his diploma in Electrical Engineering from Ilmenau University of Technology and the Ph.D. degree from the Dresden University of Applied Sciences in 1977. From 1972 to 1976 he worked as research assistant at the University and from 1976 to 1991 as graduate assistant at the Institute of Cybernetics and Information Processes of the Academy of Science of the GDR. Since 1992 he is with the Fraunhofer Institute for Integrated Circuits in the Design Automation Division. His key activities are simulation based performance analysis and optimisation, design of digital circuits, model-based design of automation systems, and development of software tools for design support. His email address is ulrich.donath@eas.iis.fraunhofer.de.

JÜRGEN HAUFE received his diploma in Electrical Engineering from Dresden University of Technology in 1976 and the Ph.D. degree from the Academy of Science of the GDR in 1987. Since 2001 he is Head of the System Simulation Group and later he became Head of the Automation System Group of the Design Automation Division EAS. At present, his main research activities are focused on model based design strategies and tools for automation systems in the field of manufacturing and building automation. His email address is juergen.haufe@eas.iis.fraunhofer.de. 